\title{
Endoscopic resection using an over-the-scope clip for duodenal neuroendocrine tumors $\square$
}

\section{(๑) $\odot \ominus$}

\author{
Authors

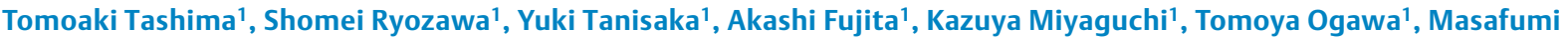 \\ Mizuide', Yumi Mashimo¹, Tomonori Kawasaki², Masami Yasuda²
}

Institutions

1 Department of Gastroenterology, Saitama Medical University International Medical Center, Saitama, Japan

2 Department of Pathology, Saitama Medical University International Medical Center, Saitama, Japan

submitted 17.9.2020

accepted after revision 20.1.2021

Bibliography

Endosc Int Open 2021; 09: E659-E666

DOI 10.1055/a-1374-6141

ISSN 2364-3722

(C) 2021. The Author(s).

This is an open access article published by Thieme under the terms of the Creative Commons Attribution-NonDerivative-NonCommercial License, permitting copying and reproduction so long as the original work is given appropriate credit. Contents may not be used for commercial purposes, or adapted, remixed, transformed or built upon. (https://creativecommons.org/licenses/by-nc-nd/4.0/)

Georg Thieme Verlag KG, Rüdigerstraße 14,

70469 Stuttgart, Germany

Corresponding author

Tomoaki Tashima, MD, PhD, Department of

Gastroenterology, Saitama Medical University International Medical Center, 1397-1 Yamane, Hidaka City, Saitama 3501298, Japan

Fax: +81-42-984-4589

t.tashima1981@gmail.com

\section{ABSTRACT}

Background and study aims Endoscopic resection of duodenal neuroendocrine tumors (DNETs) remains controversial, and its indications are still unclear. This study aimed to evaluate short-term outcomes of a newly developed endoscopic muscularis resection (EMR) method that utilizes an over-the-scope clip (OTSC), termed EMRO, for treating DNETs.

Patients and methods In total, 13 consecutive patients with 14 small $(\leq 10 \mathrm{~mm})$ DNETs who underwent EMRO from September 2017 to March 2020 were retrospectively enrolled. EMRO was performed by a single experienced endoscopist. Patients' characteristics and treatment outcomes were assessed.

Results The En bloc and R0 resection rates were $100 \%$ (14/ 14 ) and $92.9 \%(13 / 14)$, respectively. The median pathological resected specimen size was $10 \mathrm{~mm}$, with a median pathological resected tumor size of $6 \mathrm{~mm}$. During the EMRO procedure, there was no occurrence of misplacement of the OTSC to the target lesion. With respect to the pathological resection depth, nine cases (64.3\%) and five cases (35.7\%) were categorized as deep submucosal resection and muscularis resection, respectively, whereas no case was categorized as full-thickness resection. There were no intraoperative or delayed perforations. However, delayed bleeding occurred in two cases. At a median follow-up of 12 months (range 7-36) after EMRO, there was no incidence of local recurrence. At the first follow-up endoscopy performed at 6 months after EMRO, the OTSC was retained in place in two of 14 DNETs (14.3\%).

Conclusions EMRO can be performed safely, by an experienced endoscopist, for small ( $\leq 10 \mathrm{~mm}$ ) DNETs.

\section{Introduction}

Duodenal neuroendocrine tumors (DNETs) are considered rare $[1,2]$, occurring less frequently than rectal and gastric neuroendocrine tumors (NETs) and accounting for $<5 \%$ of all gastrointestinal NETs [1]. Endoscopic resection of DNETs remains controversial and its indications unclear. Endoscopic mucosal resection (EMR) [3,4], cap-assisted endoscopic mucosal resec- tion (EMR-C) [3], endoscopic submucosal resection with ligation (ESMR-L) [5-7], and endoscopic submucosal dissection (ESD) $[4,8,9]$ are deemed suitable for the resection of small DNETS $(\leq 10 \mathrm{~mm}$ ) with a low malignancy potential. DNETs develop from the deep mucosa and are histologically classified as epithelial tumors and macroscopically as submucosal tumors [10]. Thus, deep layer resection is required to achieve $\mathrm{R} 0$ resection. However, endoscopic treatment of DNETs has frequently 
resulted in incomplete resection [4], and endoscopists have to consider an increased risk of perforation when attempting to achieve an ample vertical safety margin in the thin duodenal wall. Therefore, an easy, safe, and reliable endoscopic resection procedure is needed for such lesions.

Recently, the over-the-scope clip (OTSC) system (Ovesco Endoscopy $\mathrm{GmbH}$, Tübingen, Germany), a novel gastrointestinal full-thickness closure device, was reported to be safe and effective for fistula closure, anastomotic dehiscence, hemostasis of gastrointestinal bleeding, and endoscopic closure of iatrogenic gastrointestinal perforations [11]. Mucosal defect closure using the OTSC system after duodenal ESD has helped prevent the previously mentioned severe adverse events (AEs) $[12,13]$. Hence, we developed a new endoscopic resection method using the strong cerclage force of the OTSC, with the device placed prior to resection so as to surround the basal portion of the target lesion to achieve tumor resection deeper than the muscle layer and prevent intraoperative and delayed perforation. We termed this procedure endoscopic muscularis resection with OTSC (EMRO). In this study, our aim was to evaluate the short-term outcomes of EMRO for treating DNETs.

\section{Patients and methods}

\section{Study design}

This retrospective observational study was conducted at Saitama Medical University International Medical Center and performed in accordance with the Declaration of Helsinki. The study protocol was approved by our institution's ethics committee (institutional ID: 19-315) and written informed consent was obtained from all patients after the risks and benefits of the treatment had been fully explained prior to EMRO.

\section{Patients and methods}

From September 2017 to March 2020, 13 consecutive patients with 14 DNETs $\leq 10 \mathrm{~mm}$ who underwent EMRO at our institute were enrolled. The inclusion criteria were: 1 . Tumors diagnosed as NETs through preoperative pathological biopsy; 2. Lesions $\leq 10 \mathrm{~mm}$ in diameter identified on endoscopy; 3 . Lesions located within the submucosal layer; and 4. No lymph node or distant metastasis detected. Considering the risk of acute pancreatitis, we did not attempt EMRO for lesions in which OTSC deployment could involve the major papillae. We excluded patients who refused the use of their resected tissue samples and clinical data for this study. All 14 EMRO procedures were performed by a single endoscopist (T.T.), who had performed $>250$ duodenal endoscopic resections and >200 OTSC procedures. Information on patient and clinical characteristics, including sex, age, tumor characteristics (location, size), shortterm treatment outcomes, and follow-up outcomes (local recurrence and OTSC retainment), was retrospectively assessed.

\section{Preoperative endoscopic examination including simulation of OTSC deployment}

Before considering EMRO, all patients underwent preoperative endoscopy using a therapeutic endoscope (GIF-Q260) or GIFH290T; Olympus, Medical Systems Co., Tokyo, Japan). The lesion size, location, and position relative to the major papilla, and scope maneuverability were evaluated. Moreover, all patients underwent endoscopic ultrasonography to evaluate the depth of invasion ( $\triangleright$ Fig. 1) and computed tomography (CT) to determine the presence of lymph node and/or distant metastasis.

The most critical step in the EMRO procedure is to avoid OTSC misplacement on the target lesion. OTSC misplacement was defined as deployment of the OTSC just onto the target lesion during the EMRO procedure. To avoid misplacement, we performed simulation of OTSC deployment during the preoperative endoscopic examination for all tumors deemed to be potential candidates for EMRO. The simulation was conducted as follows. First, an attachment cap (D-201-11804; Olympus) was mounted onto the tip of the endoscope so that the total length of the endoscope with the attachment cap was roughly similar to that of the endoscope with the applicator cap when the OTSC was mounted onto the endoscope tip. Second, we confirmed that the entire target tumor could be suctioned in-
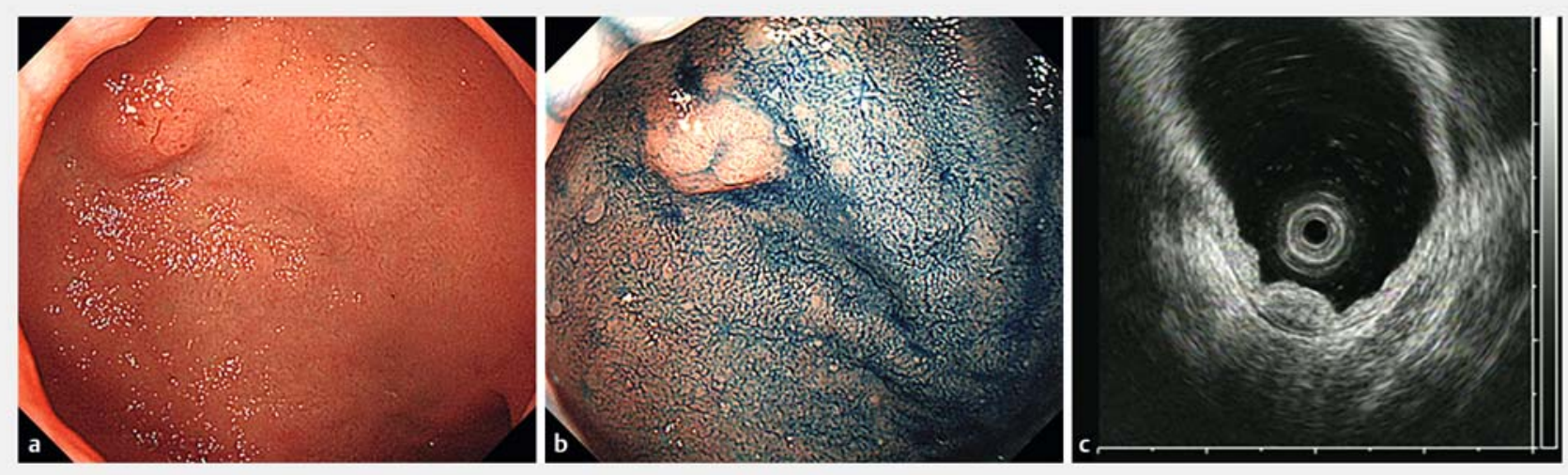

Fig. 1 Preoperative endoscopic examination before considering EMRO. a submucosal tumor measuring 5 mm, located in the duodenal bulb. b Indigocarmine staining revealing scarring from a previous biopsy in the center of the lesion. $\mathbf{c}$ Endoscopic ultrasonography imaging showing a low echoic tumor located in the third layer. EMRO, endoscopic muscularis resection with over-the-scope clip 

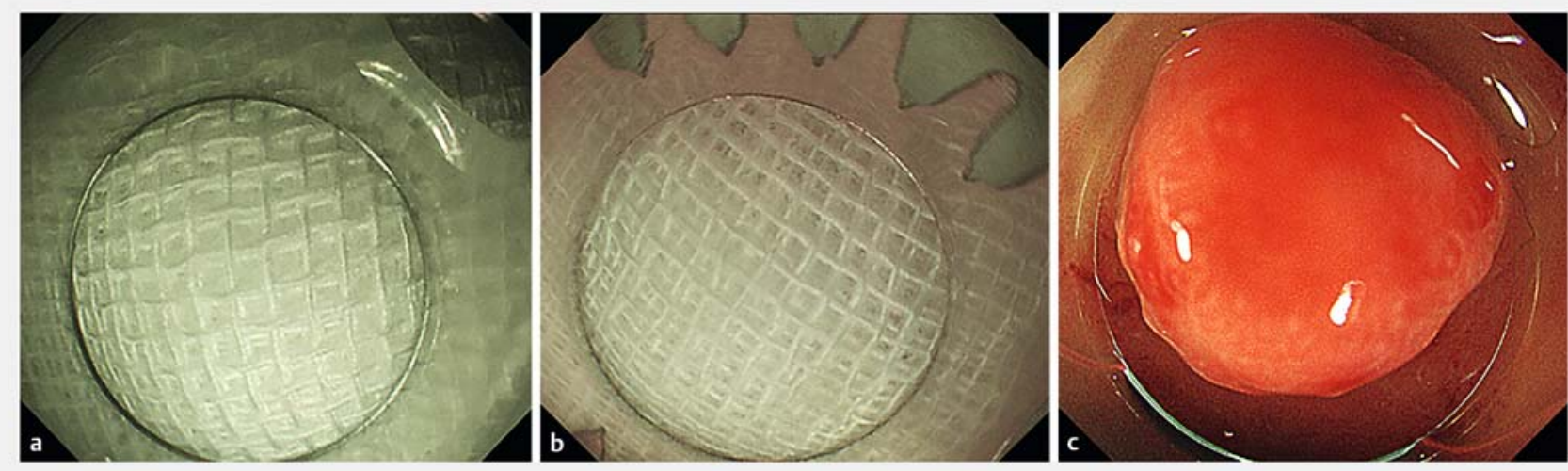

- Fig. 2 Simulation of OTSC deployment. a Image of the tip of the endoscope with a clear attachment cap. The length of the cap was adjusted and fixed such that the length from the tip of the endoscope to the cap $(6 \mathrm{~mm})$ was roughly the same as the length from the tip of the endoscope to the tip of the applicator cap when an OTSC was mounted to the tip of the endoscope. $\mathbf{b}$ Image of the tip of the endoscope, with a $9 \mathrm{t}$ $(11 / 6 \mathrm{t})$ OTSC applicator cap. c Successful simulation of OTSC deployment. The image shows that the entire target tumor could be suctioned inside the clear attachment cap. OTSC, over-the-scope clip.

side the attachment cap. We estimated the possibility and difficulty of en bloc resection of the lesion using EMRO ( $\vee$ Fig.2).

All patients had been referred from other hospitals, with preoperative biopsy completed prior to referral to our institution, with the histology of preoperative biopsies reviewed only by pathologists at the referring hospital. Histopathological assessments of resected specimens were performed by two experienced pathologists, according to the classification system of the World Health Organization [14]. In addition, immunohistochemical analysis, using chromogranin $\mathrm{A}$ and synaptophysin as neuroendocrine markers, was performed for accurate diagnosis; classification of tumors as G1, G2, or G3 was determined using the Ki-67 index.

\section{EMRO procedure}

All procedures were performed in an endoscopy room, with the patient under conscious sedation. EMRO procedures were performed using the same therapeutic endoscope as in preoperative endoscopy. All procedures were performed using a highfrequency electrosurgical unit (VIO300D; Erbe Elektromedizin, Tübingen, Germany). First, the target lesion border was marked using the tip of a 10-mm snare (Captivator II; Boston Scientific, Marlborough, Massachusetts, United States) in the soft coagulation mode (effect 3, 40W). Regarding the types of OTSCs used in the EMRO procedure, the traumatic (t) type for organs with thin walls was introduced. Then, the upper gastrointestinal endoscope was mounted with a $9 \mathrm{t}(11 / 6 \mathrm{t})$ OTSC ( $9 \mathrm{t}$, Japanese notation; $11 / 6 \mathrm{t}$, overseas notation). The lesion, including the markings, was sufficiently suctioned into the applicator cap. The OTSC was successfully deployed outside the markings, creating a target lesion pseudo-polyp. Finally, the lesion was resected en bloc above the OTSC with a snare, using the endocut mode (endocut $Q$, effect 3 , duration 1 , interval 3 ). Hemostasis for procedural or delayed bleeding was attempted using the tip of snare or hemostatic forceps in the soft coagulation mode (effect $4,60 \mathrm{~W}$ ). On the second day after the EMRO procedure, all patients routinely underwent a second endoscopy to detect any delayed bleeding or perforation ( $\vee$ Fig. $3, \triangleright$ Video 1 ).

\section{Follow-up}

All patients were hospitalized after EMRO for 2 days according to the routine followed in our institution. Regarding the protocol criteria, oral intake of regular food was resumed on the day after EMRO. Proton pump inhibitors (rabeprazole $20 \mathrm{mg} /$ day, lansoprazole $30 \mathrm{mg} /$ day, or esomeprazole $20 \mathrm{mg} /$ day) were administered for 2 weeks starting on the day of the procedure. To evaluate recurrences, patients underwent a first follow-up endoscopy at 6 months after EMRO. Thereafter, endoscopic follow-up was performed at 12 -month intervals. If a residual tumor was suspected, a biopsy was performed, and the tissue obtained was examined histologically.

\section{Outcome measurements}

Primary outcomes were the rates of en bloc and R0 resection (defined as when both the lateral and vertical margins of the specimen were found to be histologically free of tumor cells), prevalence of post-EMRO AEs, as well as intraoperative perforation, delayed perforation, and delayed bleeding rates. Delayed bleeding was diagnosed as overt bleeding occurring within 14 days after EMRO and requiring an endoscopic hemostatic procedure using hemostatic forceps. Secondary outcomes were procedure time (defined as the time from when the endoscope with OTSC was inserted into the patient's body to when the endoscope was removed); number of OTSC misplacement cases; pathological resected specimen/tumor size; invasion depth of tumor; pathological tumor type as G1, G2, or G3; and pathological resection depth. Theoretically, the resection depth achievable with EMRO can be classified into three categories according to differences in the layers of the resected gastrointestinal wall. Resection with a vertical margin including a substantial portion of the submucosal layer was termed deep submucosal resection (DSMR); resection with a vertical margin 

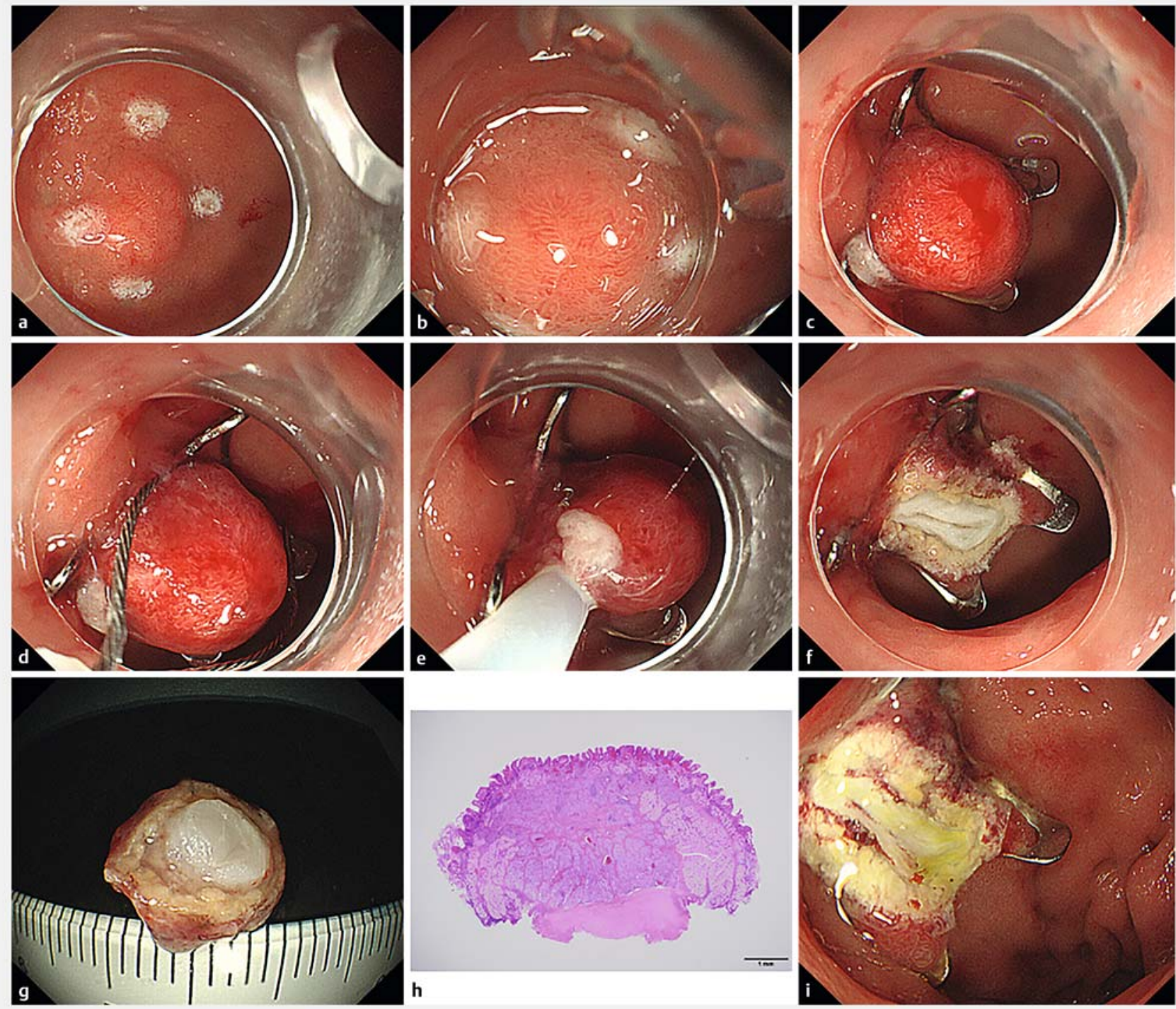

- Fig. 3 EMRO strategy for a representative case. a Four markings were created outside, but as close as possible, to the demarcation line using the tip of a snare. $\mathbf{b}$ A $9 \mathrm{t}(11 / 6 \mathrm{t})$ OTSC is mounted on the tip of the endoscope and the lesion, including all markings, is suctioned sufficiently into the applicator cap. $\mathbf{c}$ Formation of pseudo-protruding polyps after deployment of an OTSC around the tumor. $\mathbf{d}$, $\mathbf{e}$ The lesion is resected en bloc above the OTSC, with a 10-mm snare, using the endocut mode on the high-frequency electrosurgical unit. $\mathrm{f}$ Muscularis resection defect without perforation or bleeding. $\mathbf{g}$ Undersurface view of the en bloc-resected specimen with the muscle layer. $\mathbf{h}$ Histopathological examination of the resected specimen with a loupe revealing the nature of the tumor as a duodenal G1 NET with negative resection margins. The presence of the muscle layer in the vertical margin is confirmed (hematoxylin and eosin, original magnification $\times 20$ ). i Resection defect on the second postoperative day. NET, neuroendocrine tumor; EMRO, endoscopic muscularis resection with over-the-scope clip; OTSC, over-thescope clip.

including the muscle layer was referred to as muscularis resection (MR); and resection with a vertical margin, including the serosa, was termed full-thickness resection (FTR). All specimens were subjected to pathological examinations. Quantitative data are expressed as medians and interquartile ranges. All analyses were performed using SPSS, version 11.0 (SPSS Inc., Chicago, Illinois, United States).

\section{Results}

\section{Patient and lesion characteristics}

All 13 patients ( 7 men; median age, 74 years) with 14 DNETs $\leq 10 \mathrm{~mm}$ underwent EMRO during the study period. None of the patients were taking antithrombotic agents.

The patients' clinicopathological and lesion characteristics are summarized in $\mathbf{r}$ Table $\mathbf{1}$. Tumors were predominantly located in the bulb $(10 / 14,71.4 \%)$. The median tumor size was $6 \mathrm{~mm}$ (range 4 to 10 ). 


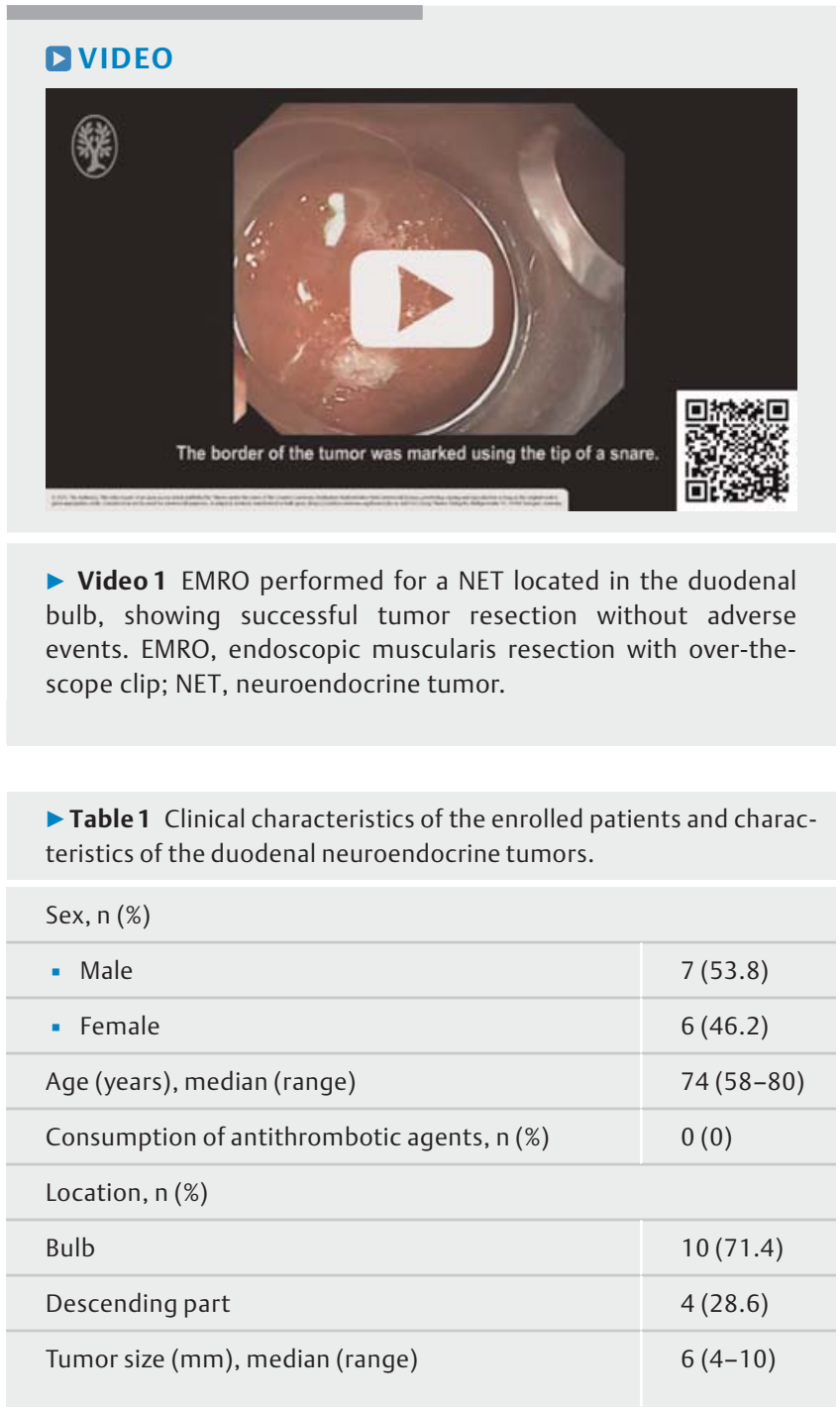

\section{Therapeutic outcomes}

The treatment outcomes of 14 DNETs are summarized in $\downarrow$ Table 2. The median procedure time was 15 minutes (range 10 to 18). During EMRO, no OTSC misplacements in target lesions occurred. The median pathological resected specimen size was $10 \mathrm{~mm}$ (range 8 to 11 ), and the median pathological resected tumor size was $6 \mathrm{~mm}$ (range 3 to 8 ).

The final pathological diagnosis of tumors revealed that all lesions invaded the submucosal layer, with a histological diagnosis of NET G1 for all 14 lesions. En bloc and R0 resection rates were $100 \%(14 / 14)$ and $92.9 \%(13 / 14)$, respectively. In one case in which $\mathrm{R} 0$ resection could not be achieved, the vertical margin was pathologically diagnosed as inconclusive. Another case had lymphovascular invasion and, thus, additional surgical resection was recommended. However, the patient insisted on continued observation. We, therefore, decided to carefully perform a follow-up endoscopy and abdominal CT every 6 months. No local recurrence or distant metastasis was noted during the 36 months after resection.

With respect to the pathological resection depth, five cases (64.3\%, 9/14) were categorized as DSMR and five cases (35.7\%,
- Table 2 Short-term outcomes of EMRO for duodenal neuroendocrine tumors.

Procedure time (min), median (range)

$15(10-18)$

OTSC misplacement, $\mathrm{n}(\%)$

$0(0)$

Pathological resected specimen size ( $\mathrm{mm})$, median

$10(8-11)$ (range)

\begin{tabular}{|l|l|}
\hline Pathological tumor size $(\mathrm{mm})$, median (range) & $6(3-8)$ \\
\hline Invasion depth, $\mathrm{n}(\%)$ & $14(100)$ \\
\hline Submucosal layer & \\
\hline Pathological type, $\mathrm{n}(\%)$ & $14(100)$ \\
\hline NET G1 & $14 / 14(100)$ \\
\hline En bloc resection rate, $\mathrm{n}(\%)$ & $13 / 14(92.9)$ \\
\hline R0 resection rate, $\mathrm{n}(\%)$ & $0(0)$ \\
\hline Lateral margin positive, $\mathrm{n}(\%)$ & $0(0)$ \\
\hline Vertical margin positive, $\mathrm{n}(\%)$ & $1(7.1)$ \\
\hline Inconclusive, $\mathrm{n}(\%)$ & $1(7.1)$ \\
\hline Lymphovascular invasion, $\mathrm{n}(\%)$ & \\
\hline
\end{tabular}

Pathological resection depth, $\mathrm{n}(\%)$

\begin{tabular}{|l|l|}
\hline Deep submucosal resection & $9(64.3)$ \\
\hline Muscularis resection & $5(35.7)$ \\
\hline Full-thickness resection & $0(0)$ \\
\hline Intraoperative perforation, $\mathrm{n}(\%)$ & $0(0)$ \\
\hline Delayed perforation, $\mathrm{n}(\%)$ & $0(0)$ \\
\hline Delayed bleeding, $\mathrm{n}(\%)$ & $2(14.3)$ \\
\hline Follow-up period (months), median (range) & $12(7-36)$ \\
\hline $\begin{array}{l}\text { Patients who underwent first follow-up endoscopy, } \\
\mathrm{n}(\%)\end{array}$ & $13(100)$ \\
\hline Local recurrence, $\mathrm{n}(\%)$ & $0(0)$ \\
\hline OTSC retainment in place at 6 months, $\mathrm{n}(\%)$ & $2(14.3)$ \\
\hline
\end{tabular}

R0 resection was achieved when both the lateral and vertical margins of the specimen were found to be histologically free of tumor cells.

Procedure time was defined as the time from insertion of the endoscope with OTSC into the patient's body to retrieval of the endoscope.

OTSC misplacement was defined as deployment of the OTSC just onto target lesions, not outside the lesions, during the EMRO procedure.

Final pathological assessments were performed according to the classification system of the World Health Organization.

Delayed bleeding was diagnosed as overt bleeding occurring within 14 days after EMRO and requiring an endoscopic hemostatic procedure using hemostatic forceps. Patients underwent a first follow-up endoscopy at 6 months after EMRO. Thereafter, endoscopic follow-up was performed at 12-month intervals. If residual tumor was suspected, a biopsy was performed, and the tissue obtained was examined histologically. EMRO, endoscopic muscularis resection with OTSC; NET, neuroendocrine tumor; OTSC, over-the-scope clip

5/14) cases were categorized as MR ( $\triangleright$ Fig. 4), whereas no case was categorized as FTR. The aforementioned case wherein the vertical margin was pathologically diagnosed as inconclusive was categorized as DSMR. 


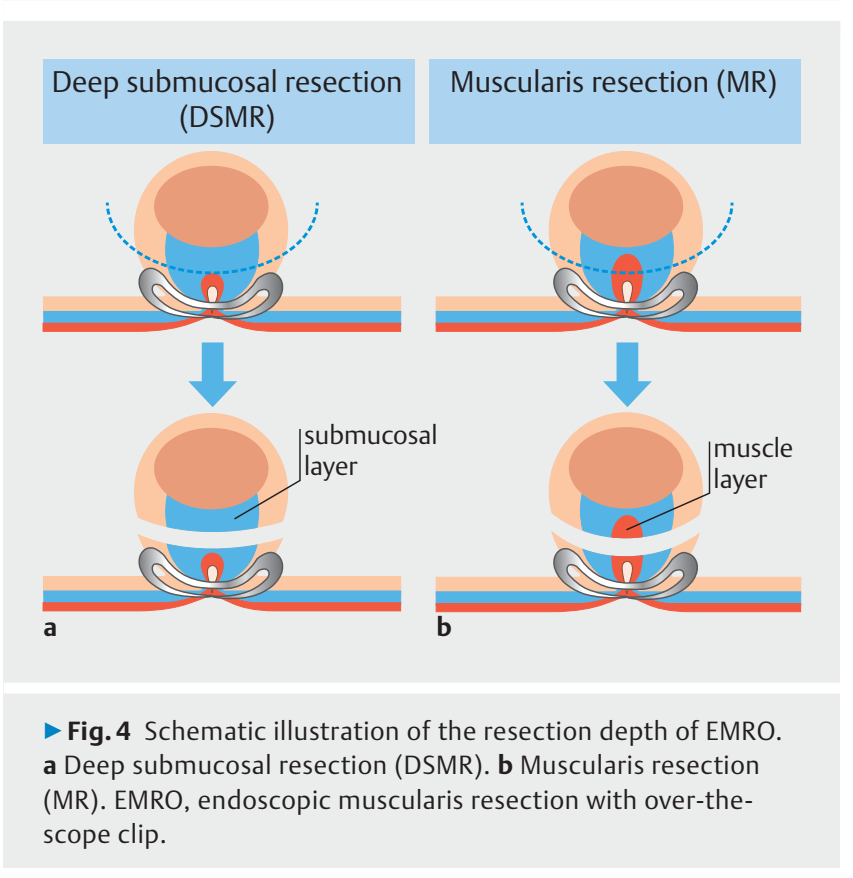

There was no incidence of intraoperative or delayed perforation. Moreover, delayed bleeding occurred in two (14.3\%, 2/14) patients, which was detected during the second endoscopic examination performed on the second day after EMRO. Both of these patients had no symptoms and bleeding in the mucosal defect within the OTSC was easily controlled by endoscopic coagulation using hemostatic forceps.

Follow-up endoscopy was performed in all patients. At a median follow-up period of 12 months (range 7 to 36) after EMRO, there was no incidence of local recurrence. On the first followup endoscopy performed at 6 months after EMRO, the OTSC was retained in place in two of 14 (14.3\%) lesions.

\section{Discussion}

Endoscopic resection combined with OTSC is a new technique. Al-Bawardy et al. [15] reported only three cases and Sarker et al. [16] and Kappelle et al. [17] reported only four cases of DNETs each. Therefore, to the best of our knowledge, our study comprises the largest number of DNETs treated with OTSC reported to date.

Among the several endoscopic resections for DNETs reported, ESMR-L [5-7] and ESD [4, 8, 9] are the mainly used standard methods. In general, ESMR-L is considered technically easy, safe, and inexpensive. Moreover, most of the endoscopists get used to performing ESMR-L in rectal NETs $[18,19]$. In terms of technical ease, implementation of EMRO for DNETs requires a learning curve. EMRO for DNETs should be performed by an endoscopist who is experienced in duodenal endoscopic resection and OTSC procedures. However, perforation has even been reported in patients treated with ESMR-L [7]. Furthermore, ESD for DNETs takes more time [4,9] and is challenging because of the high risk of perforation [9]. When comparing EMRO to the above duodenal endoscopic resection techniques in terms of $\mathrm{R} 0$ resection rate and $\mathrm{AEs}$, the $\mathrm{R} 0$ resection rate was $92.9 \%$ and the intraoperative or delayed perforation rate was $0 \%$, suggesting that EMRO considerably reduces intraoperative and delayed perforation risks while achieving relatively higher $\mathrm{R} 0$ resection rates. However, EMRO is still an inadequate technique for securing the vertical margin because FTR was not achieved in any case and MR was achieved in only $35.7 \%$ of cases. Similarly, Sarker et al. reported that FTR was not achieved in any patient with DNET [16]. On the other hand, we previously reported a case of rectal NET, wherein FTR was achieved by EMRO [20]. The EMRO procedure was consistently performed for all cases and the operator could not adjust the resection depth. Based on our results, it would be extremely difficult to achieve FTR by EMRO in the duodenum; thus, to reliably achieve RO resection, it is reasonable to target tumors that are expected to be located within the deepest layer of the submucosa. However, in the present study, the case in which the vertical margin was pathologically diagnosed as inconclusive was resected with a vertical margin, including a deep submucosal layer. Therefore, further device and technical modifications (i.e., the shape of the clip and snare, depth of the cap, etc.) for achieving at least MR at a high rate are necessary in the near future.

In our study, there were no intraoperative and delayed perforations and all patients were discharged from the hospital on post-EMRO Day 2. The short hospital stay is an important advantage of EMRO. However, Kappelle et al. [17] reported that one case of perforation occurred after endoscopic full-thickness resection (EFTR) using a different type of OTSC than the one we used in this study. Because perforation can occur in EMRO, it is necessary to cut the lesion instantly using the endocut mode, being careful not to contact or get caught in the OTSC during snaring.

Cases of delayed bleeding (2/14 cases, $14.3 \%$ ) can be easily managed with endoscopic coagulation ( $\triangleright$ Fig.5). However, the delayed bleeding rate in our study was higher than the previously reported rates for ESMR-L [5-7] and ESD [4, 8, 9]. Therefore, when performing prophylactic endoscopic coagulation of a mucosal defect to the fullest extent possible, even without bleeding immediately after resection, it is recommended that surgeons always perform a second endoscopy on the next day after EMRO. Nevertheless, because the OTSC is made from a nitinol-based metal, it is important to consider that coagulation can conduct heat to the OTSC, resulting in delayed perforation due to thermal damage to the duodenal mucosa. Fortunately, no cases of delayed perforation were observed after endoscopic treatment using OTSC with coagulation, in the present study, or in our previous report [12] or any other reports. However, from a safety perspective, coagulation should be kept to the minimum required and direct coagulation with the OTSC and at high-frequency setting should be avoided. Otherwise, additional clipping of the mucosal defect within the OTSC after EMRO is also considered one of the methods to prevent delayed bleeding. However, we have tried it in several cases, but it failed because the clips were repelled by the OTSC.

Recently, a dedicated FTR device (FTRD; Ovesco Endoscopy AG) was made commercially available for mainly colorectal EFTR in Europe and the United States [21-23]. However, this device has not yet been approved for use in Japan. Thus, we 

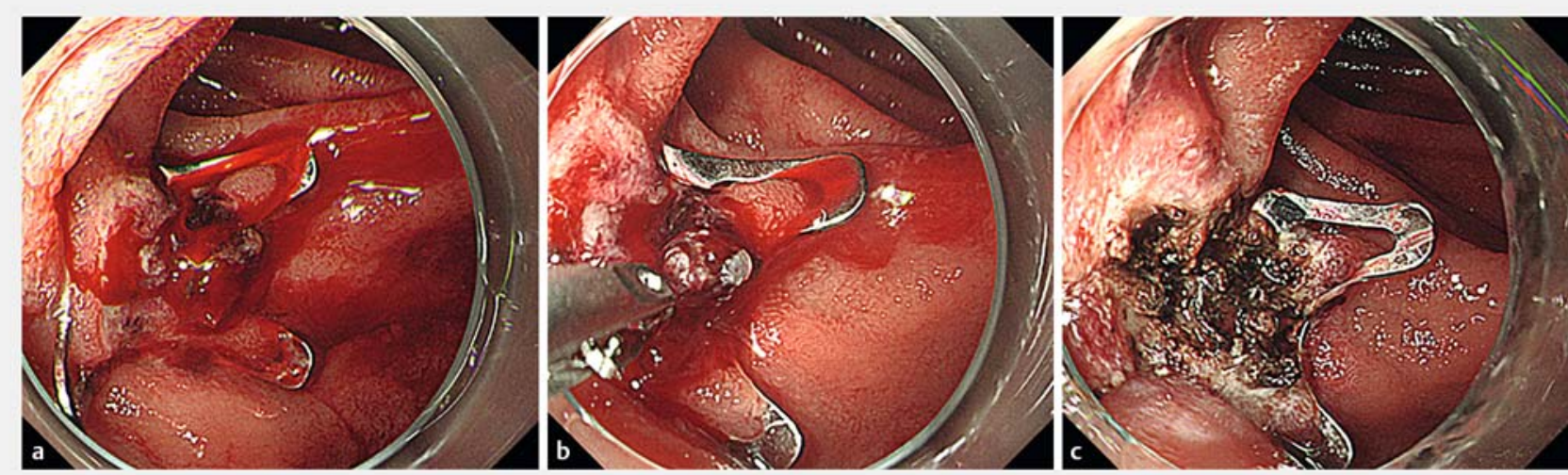

- Fig. 5 Hemostasis for delayed bleeding after EMRO. a Delayed bleeding in the mucosal defect within the OTSC on the second postoperative day in this case. b, $\mathbf{c}$ Endoscopic images showing complete control of the bleeding using hemostatic forceps, without perforation. EMRO, endoscopic muscularis resection with over-the-scope clip; OTSC, over-the-scope clip.

could not consider FTRD and used a commercially available OTSC for endoscopic resection of the duodenal lesions. In the meta-analysis by Brewer et al. regarding FTR using OTSC and FTRD, a high overall R0 resection rate was observed (81\% for upper gastroinestinal subepithelial lesions) [24]. Similarly, in our study, the R0 resection rate was $92.9 \%$. Thus, EMRO has been shown to be available for endoscopic resection of subepithelial lesions, such as DNETs.

The EMRO also has some disadvantages. First, the cost-effectiveness should be considered. A single OTSC costs approximately $\$ 800$ or $€ 600$. Therefore, EMRO costs more than other conventional endoscopic resections. Currently, the use of OTSC for endoscopic resection is off-label, at least in Japan. Therefore, the medical fee points are calculated as a conventional EMR for duodenal tumors and the cost of the OTSC is paid by the hospital. Second, once an OTSC is placed inappropriately, such as just onto the target lesion, it is difficult to continue the resection. In our study, there were no OTSC misplacements. Currently, some OTSC removal methods, using clip cutting devices, have been reported, including: argon plasma coagulation [25]; the Nd:YAG laser [26]; and the remOVE system (DC Cutter; Ovesco Endoscopy) [27]. Among the three cutting devices, the remOVE system has been reported to be the safest and most effective in the clinical studies. However, this device has not yet been approved for use in Japan. It is expected to be introduced in clinical practice as soon as possible. Use of other removal methods, such as grasping forceps [28], EMR/ESD [29] and ice-cold saline solution [30], also have been reported. The safety and efficacy of these methods are uncertain because they have been described in only a few cases; moreover, the use of these methods is not recommended because they can cause new severe AEs [31]. Considering the above, intentional removal of an OTSC is not realistic; for this reason, sufficient preoperative simulation of OTSC deployment is recommended, as well as limitation of the target to only lesions in which the OTSC can be reliably deployed. Third, it is technically very difficult to perform repeat endoscopic resection of residual and recurrent lesions that are located within the retained OTSC. In our study, the OTSC was retained in $14.3 \%$ of the lesions. If the OTSC is removed naturally or by the aforementioned removal methods, residual and recurrent lesions can be resected with EMRO [32]; if they cannot be removed, surgical resection may be considered [21].

The limitations of our study should be acknowledged. First, this was a single-center study with a small sample size, retrospective design, and lack of a control group, which might have introduced a selection bias. Second, all procedures were performed by a single experienced endoscopist and the outcomes could not be directly generalized. Third, this study had a short follow-up period and, thus, long-term follow-up outcomes are not available. Thus, prospective, multicenter, randomized controlled trials with larger sample sizes are warranted to establish the clinical usefulness of EMRO, to fully confirm its safety and effectiveness, and to investigate its long-term outcomes.

\section{Conclusion}

EMRO can be performed safely by an experienced endoscopist on small DNETs $(\leq 10 \mathrm{~mm})$. Further comparative studies are required to evaluate the safety and efficacy of EMRO compared to other endoscopic resection techniques.

\section{Acknowledgements}

The authors thank Editage (www.editage.com) for English language editing.

\section{Competing interests}

The authors declare that they have no conflict of interest.

\section{References}

[1] Modlin IM, Lye KD, Kidd M. A 5-decade analysis of 13,715 carcinoid tumors. Cancer 2003; 97: 934-959 
[2] Yao JC, Hassan M, Phan A et al. One hundred years after "carcinoid": epidemiology of and prognostic factors for neuroendocrine tumors in 35,825 cases in the United States. J Clin Oncol 2008; 26: 3063-3072

[3] Gincul R, Ponchon T, Napoleon B et al. Endoscopic treatment of sporadic small duodenal and ampullary neuroendocrine tumors. Endoscopy 2016; 48: 979-986

[4] Kim GH, Kim Jl, Jeon SW et al. Endoscopic resection for duodenal carcinoid tumors: a multicenter, retrospective study. J Gastroenterol Hepatol 2014; 29: 318-324

[5] Kim TW, Kim GH, Park DY et al. Endoscopic resection for duodenal subepithelial tumors: a single-center experience. Surg Endosc 2017; 31: 1936-1946

[6] Oono Y, Shinmura K, Hori K et al. Endoscopic submucosal resection using a ligation device without injection for duodenal neuroendocrine tumors. Surg Endosc 2019; 33: 2008-2014

[7] Fujimoto A, Sasaki M, Goto O et al. Treatment results of endoscopic mucosal resection with a ligation device for duodenal neuroendocrine tumors. Intern Med 2019; 58: 773-777

[8] Matsumoto S, Miyatani H, Yoshida Y. Endoscopic submucosal dissection for duodenal tumors: a single-center experience. Endoscopy 2013; 45: 136-137

[9] Nishio M, Hirasawa K, Ozeki Y et al. Short- and long-term outcomes of endoscopic submucosal dissection for non-ampullary duodenal neuroendocrine tumors. Ann Gastroenterol 2020; 33: 265-2671

[10] Sato Y, Hashimoto S, Mizuno K et al. Management of gastric and duodenal neuroendocrine tumors. World J Gastroenterol 2016; 22: 6817-6828

[11] Kobara H, Mori H, Nishiyama $\mathrm{N}$ et al. Over-the-scope clip system: A review of 1517 cases over 9 years. J Gastroenterol Hepatol 2019; 34: 22-30

[12] Tashima T, Ohata K, Sakai E et al. Efficacy of an over-the-scope clip for preventing adverse events after duodenal endoscopic submucosal dissection: a prospective interventional study. Endoscopy 2018; 50 : 487-496

[13] Dohi O, Yoshida N, Naito Y et al. Efficacy and safety of endoscopic submucosal dissection using a scissors-type knife with prophylactic over-the-scope clip closure for superficial non-ampullary duodenal epithelial tumors. Dig Endosc 2020; 32: 904-913

[14] Nagtegaal ID, Odze RD, Klimstra D et al. The 2019 WHO classification of tumours of the digestive system. Histopathology 2020; 76: 182188

[15] Al-Bawardy B, Rajan E, Wong Kee Song LM. Over-the-scope clip-assisted endoscopic full-thickness resection of epithelial and subepithelial Gl lesions. Gastrointest Endosc 2017; 85: 1087-1092

[16] Sarker S, Gutierrez JP, Council L et al. Over-the-scope clip-assisted method for resection of full-thickness submucosal lesions of the gastrointestinal tract. Endoscopy 2014; 46: 758-761
[17] Kappelle WFW, Backes Y, Valk GD et al. Endoscopic full-thickness resection of gastric and duodenal subepithelial lesions using a new, flatbased over-the-scope clip. Surg Endosc 2018; 32: 2839-2346

[18] Kwon MJ, Kang HS, Soh JS et al. Lymphovascular invasion in more than one-quarter of small rectal neuroendocrine tumors. World J Gastroenterol 2016; 22: 9400-9410

[19] Bang BW, Park JS, Kim HK et al. Endoscopic resection for small rectal neuroendocrine tumors: Comparison of endoscopic submucosal resection with band ligation and endoscopic submucosal dissection. Gastroenterol Res Pract 2016: doi:10.1155/2016/6198927

[20] Tashima T, Nonaka K, Ryozawa S. Successful endoscopic en bloc fullthickness and complete resection for two adjacent rectal neuroendocrine tumors. Dig Endosc 2019; 31: 592

[21] Schmidt A, Beyna T, Schumacher B et al. Colonoscopic full-thickness resection using an over-the-scope device: a prospective multicentre study in various indications. Gut 2018; 67: 1280-1289

[22] Rajan E, Wong Kee Song LM. Endoscopic full thickness resection. Gastroenterology 2018; 154: 1925-37.e2

[23] Bauder M, Schmidt A, Caca K. Endoscopic full-thickness resection of duodenal lesions-a retrospective analysis of 20 FTRD cases. United European Gastroenterol J 2018; 6: 1015-1021

[24] Brewer Gutierrez OI, Akshintala VS, Ichkhanian Y et al. Endoscopic full-thickness resection using a clip non-exposed method for gastrointestinal tract lesions: a meta-analysis. Endosc Int Open 2020; 8: E313-E325

[25] Kapadia S, Nagula S, Kumta NA. Argon plasma coagulation for successful fragmentation and removal of an over-the-scope clip. Dig Endosc 2017; 29: 820-821

[26] Fähndrich M, Sandmann M, Heike M. Removal of over the scope clips (OTSC) with an Nd:YAG Laser. Z Gastroenterol 2011; 49: 579-583

[27] Schmidt A, Riecken B, Damm M et al. Endoscopic removal of over-thescope clips using a novel cutting device: a retrospective case series. Endoscopy 2014; 46: 762-766

[28] von Renteln D, Denzer UW, Schachschal G et al. Endoscopic closure of GI fistulae by using an over-the-scope clip (with videos). Gastrointest Endosc 2010; 72: 1289-1296

[29] Mumtaz S, Appannagari A, Gupta N. Two endoscopic resection methods for the removal of an over-the-scope clip. Gastrointest Endosc 2015; 82: 744

[30] Krishna SG, Shakhatreh M. Endoscopic removal of over-the-scope clip with cold saline solution technique. Gastrointest Endosc 2016; 84: 850-851

[31] Ou YH, Kong WF, Li LF et al. Methods for endoscopic removal of overthe-scope clip: A systematic review. Can J Gastroenterol Hepatol 2020: doi: $10.1155 / 2020 / 5716981$

[32] Tashima T, Nonaka K, Ryozawa S. Successful further endoscopic mucosal resection with an over-the-scope clip with circumferential mucosal incision for a residual rectal neuroendocrine tumor. Dig Endosc 2020; 32: 629 